\title{
FREE SIALIC ACIDS IN THE SEMINAL VESICLE SECRETION OF THE GOLDEN HAMSTER
}

\author{
J. P. FOUQUET \\ Laboratoire de Biologie cellulaire et Centre de Physiologie cellulaire, Université Paris VI, \\ 7 Quai Saint-Bernard, 75 Paris Vème, France
}

(Received 23rd June 1971, accepted 10th September 1971)

Preliminary observations of the carbohydrate content of the male accessory organs and their secretions in the golden hamster have shown (Fouquet, 1969) that the seminal vesicles and the different lobes of the prostate are devoid of fructose and sorbitol. This contrasts with the finding in most other mammals (Mann, 1964). On the other hand, all glands secrete a small amount of free glucose and contain approximately the same quantity of mesoinositol. The presence of glucose, chiefly in the prostatic secretions, is not an artefact arising from the breakdown of tissue glycogen during either dissection or extraction, as the glandular cells lack the polysaccharide.

Among other unidentified products, there was a large amount of apparently free sialic acid(s), mainly in the vesicular secretion. In fact, the ethanolic extracts subjected to the colorimetric procedures of Warren (1959) and Roe (1934), gave the same chromophores as $\mathrm{N}$-acetylneuraminic acid (NANA) and $\mathrm{N}$-glycolylneuraminic acid (NGNA), with a peak at $549 \mathrm{~m} \mu$ in the first reaction and maximum absorbence between 465 and $470 \mathrm{~m} \mu$ in the second reaction. Moreover, on chromatograms of those extracts run with varying solvent-systems (Fouquet, 1969), there was always a large spot identified either as pink-purple by the HCl-urea reagent for ketosugars (Dedonder, 1952) or red by the thiobarbituric acid reagent for sialic acids (Warren, 1960). Using the same reagents, only NANA and NGNA stained as this unknown compound among many other carbohydrates tested, including polyols, free sugars, phosphosugars and aminosugars. However, the rate of migration of the product was different from those of the two sialic acids tested; it was decided, therefore, to reinvestigate the question.

Previous results were confirmed not only with ethanolic extracts, but also with extracts prepared with perchloric acid, trichloroacetic acid and with a mixture of barium hydroxide and zinc sulphate. Analysis of crude ethanolic extracts by Roe's procedure as well as by Warren's method gave comparable results: $100 \mathrm{ml}$ vesicular secretion were found to contain 980 to $1150 \mathrm{mg}$ free sialic acids (range values from determinations on twenty different samples, with NANA as standard). Often, with the other extracts, especially those prepared with acids, slightly higher values were obtained, probably due to moderate hydrolysis of muco-proteins and liberation of additive sialic acids and interfering substances. For that reason, ethanolic extracts, obtained by 
addition of two to three volumes of absolute ethanol to one volume vesicular secretion, were preferred for further analysis.

The ethanol-precipitable portion subjected to hydrolysis in $0.1 \mathrm{~N}$-sulphuric acid for $1 \mathrm{hr}$ at $80^{\circ} \mathrm{G}$, was found to contain about $40 \mathrm{mg}$ bound sialic acids $/ 100$ $\mathrm{ml}$ secretion (chiefly NANA). The ethanol-soluble portion containing about $1100 \mathrm{mg}$ free sialic acids/100 ml secretion was firstly chromatographed overnight on Whatman No. 1 paper with water-saturated phenol which had been previously found to separate three products stained as authentic sialic acids and giving the same chromophores with Warren's and Roe's procedures. One of them, with a very low rate of migration, seemed to be a sialyloside, accounting only for 2 to $3 \%$ of the total amount of free sialic acids. Another one, with a high rate of migration and accounting for 10 to $15 \%$, could be a di- or possibly a tri-acetylneuraminic acid. The most important product $(\mathrm{X})$, intercalated between the two others, accounted for 85 to $90 \%$ of the free sialic acids; it was revealed not only by $\mathrm{HCl}$-urea and thiobarbituric acid but also by the Erlich spraying reagent (see in Gottschalk, 1960). This X product was subsequently purified for further identification.

In a typical experiment, the starting material was about $10 \mathrm{ml}$ vesicular secretion. The concentrated ethanolic extract was chromatographed over-night in the phenolic solvent and the strip of paper was immersed in ether in order to remove phenol. After elution with $50 \%$ ethanol and evaporation at room temperature, the $\mathrm{X}$ product was chromatographed a second time over a period of 4 days with butanol-water (86/14); this step eliminated glucose and some amino-acids. A third chromatography in n-butanol, n-propanol, and $0 \cdot 1$ $\mathrm{N}$-HCl $(1 / 2 / 1)$ overnight, gave a complete separation of the free sialic acid (X) from a remaining hexitol and traces of a saccharide. The final product eluted with $50 \%$ ethanol and evaporated at room temperature consisted of 40 $\mathrm{mg}$ of a crystalline yellowish substance which became brownish after a few days.

At that time, the chromatographic behaviour of the presumed free sialic acid (X) was practically the same as that of synthetic NANA (Sigma) in a different solvent-system. The $R_{F}$ values were 0.47 in the last solvent and 0.40 in water-saturated phenol. The discrepancy with our preliminary results (Fouquet, 1969) is probably due to the complex composition of the crude extracts. Moreover, the infra-red spectrum (Kbr pellet) of the $\mathrm{X}$ product could be superimposed on the infra-red spectrum of authentic NANA. The nuclear magnetic resonance spectrum of $\mathrm{X}$ and that of synthetic NANA were found to be identical. Preliminary study by mass spectrometry gave relevant results.

Considering the mildness of the extraction and purification procedures, it seems that the vesicular secretion of the golden hamster contains an exceptionally high content of free $\mathrm{N}$-acetylneuraninic acid and probably two other sialyl compounds of unknown structure. Moreover, in this secretion, the sialic acidbound fraction is very small. Such a situation is surprising since not only in semen (Mann, 1964) but in most biological material too (Gottschalk, 1960), sialic acids are chiefly bound to glycoproteins or glycolipids. Of the accessory organs of five rodent species studied (Fouquet, 1971), only the seminal vesicles of the Chinese hamster were found to secrete a significant but much smaller amount of free sialic acids. The semen of the rodent, like that of the golden 
hamster, is devoid of fructose and sorbitol. The significance of the secretion of free sialic acids remains to be assessed with regard to the quality of the semen and the process of fertilization, but these compounds are not metabolized by spermatozoa.

We are indebted to $\mathrm{Dr} \mathrm{N}$. Thoai for realization and interpretation of the infra-red, nuclear magnetic resonance and mass spectra.

\section{REFERENCES}

Dedonder, R. (1952) Les glucides du topinambour. I. Mise en évidence d'une série de glucofructosanes dans les tubercules du topinambour. Isolement, analyse et structure des termes les moins polymérisés. Bull. Soc. Chim. biol. 34, 144.

Fouguet, J. P. (1969) Analyse des glucides dans les glandes accessoires mâles chez le hamster doré. C. r. hebd. Stanc. Acad. Sci., Paris D, 268, 1630.

FouQUET, J. P. (1971) Secretion of free glucose and related carbohydrates in the male accessory organs of rodents. Comp. Biochem. Physiol. 40A, 305.

Gotrschalk, A. (1960) The chemisiny and biology of sialic acids and related substances. Cambridge University Press.

Mann, T. (1964) The biochemistry of semen and of the male reproductive tract. Methuen, London.

Ros, J. H. (1934) A colorimetric method for the determination of fructose in blood and urine. F. biol. Chem. $107,15$.

Warren, L. (1959) The thiobarbituric acid assay of sialic acids. F. biol. Chem. 234, 1971.

WARREN, L. (1960) Thiobarbituric acid spray reagent for deoxysugars and sialic acids. Nature, Lond. 186, 237. 